\title{
Dobrushin Uniqueness Techniques and the Decay of Correlations in Continuum Statistical Mechanics
}

David Klein

Department of Mathematics, Louisiana State University, Baton Rouge, LA 70803, USA

\begin{abstract}
Uniqueness of Gibbs states and decay properties of averaged, two point correlation functions are proved for many-body potentials in continuum statistical mechanical models via Dobrushin uniqueness techniques.
\end{abstract}

\section{Introduction}

Gross [6], using Dobrushin uniqueness techniques [1], has given decay rates for two point correlation functions in classical lattice models at high temperature or low activity. This paper extends those techniques to the continuum case and gives sufficient conditions on physically reasonable continuum potentials for the analogous results to hold along with uniqueness of Gibbs states. The models studied here are based on the same measurable space used by Preston [12] and Ruelle [14] in their studies of Gibbs states. Our results rest on the assumption that the set of Gibbs states for the models we consider is non-empty at high temperature. This has been shown to be true in the case of pair potentials by Ruelle [14].

Section 2 of the paper extends the results of Gross [6] to the continuum case with necessary added hypotheses. Section 3 gives conditions on potentials for these hypotheses to be satisfied.

\section{Section 1. Notations and Definitions}

Let $\Lambda$ be a bounded Borel set in $\mathbb{R}^{d}$. We take $\left(X(\Lambda), B_{\Lambda}\right)$ to denote the measurable space of configurations of particles in $\Lambda$ described in Preston [12], and $X_{N}(\Lambda)$ denotes the configurations of cardinality $N$ in $\Lambda$. Let $\Omega$ be the set of locally finite subsets of $\mathbb{R}^{d}$, representing configurations of particles in $\mathbb{R}^{d}$. We will let $\Omega_{F} \subset \Omega$ denote the subsets of finite cardinality in $\Omega$ and $|s|$ denote the cardinality of $s \in \Omega_{F}$. $S$ is the $\sigma$-algebra on $\Omega$ generated by sets of the form $\{s \in \Omega:|s \cap B|=m\}$, where $B$ runs over bounded Borel sets of $\mathbb{R}^{d}$ and $m$ runs over the set of non-negative integers. 
The measurable space $(\Omega, S)$ has a natural product space structure. For each $a \in \mathbb{Z}^{d}$, let

$$
\Lambda_{a}=\left\{r \in \mathbb{R}^{d}: a^{i}-\frac{1}{2} \leqq r^{i}<a^{i}+\frac{1}{2}, i=1,2, \ldots, d\right\}
$$

The cells $\left\{\Lambda_{a}\right\}$ partition $\mathbb{R}^{d}$. Let $a_{1}, a_{2}, a_{3}, \ldots$ be an enumeration of $\mathbb{Z}^{d}$ and to simplify notation let

$$
\Lambda_{i}=\Lambda_{a_{i}}
$$

It is easy to verify (see Preston [12], Ruelle [14]) that $(\Omega, S)$ is isomorphic in a natural way to

$$
\prod_{i=1}^{\infty}\left(X\left(\Lambda_{i}\right), B_{\Lambda_{i}}\right) \text {. }
$$

We will identify these two measurable spaces throughout.

Definition 1.1. For any configuration $s \in \Omega$ and any set $\Lambda \subset \mathbb{R}^{d}$, we let $s_{\Lambda}=s \cap \Lambda$, and we write $s=s_{\Lambda} \vee \hat{s}$, where $\hat{s}=s \backslash s_{\Lambda}=S_{\mathbb{R}^{d} \backslash \Lambda^{*}}$. We also denote $s_{a}=s_{\Lambda_{a}}$.

Note that if we express $s=s_{a} \vee \hat{s}$, then the symbol " $\vee$ " may be interpreted as "union" or, with the product structure of $\Omega$, as "Cartisean product".

We will consider many-body interactions $V: \Omega_{F} \rightarrow(-\infty, \infty]$ of the form

$$
V(x)=\sum_{N=2}^{|x|} \sum_{\substack{y \subset x \\|y|=N}} \phi_{N}(y)
$$

for some potentials $\phi_{N}$. We will assume throughout that $V$ is $S$-measurable, stable, and translation invariant.

Definition 1.2. For any $x \in X(\Lambda), s \in \Omega$ such that $s \cap \Lambda=\emptyset$ and bounded Borel set $\tilde{\Lambda} \supset \Lambda$,

$$
V_{\Lambda}(x \mid s \cap \tilde{\Lambda})=\sum_{N=2}^{\infty} \sum_{\substack{y \subset x \vee s \\ y \cap x \neq \neq \\|y|=N}} \phi_{N}(y)
$$

We define the sets $R_{\Lambda}^{0}, R_{\Lambda}^{+}, R_{\Lambda}^{-}$, and $R_{\Lambda}$ in complete analogy to Preston [12, p. 97], along with the map $V_{\Lambda}(x \mid s): R_{\Lambda}^{0} \rightarrow(-\infty, \infty]$ given by

Definition 1.3. For $s \in \Omega$ such that $s \cap \Lambda=\emptyset$,

$$
V_{\Lambda}(x \mid s)=\lim _{\tilde{\Lambda} \uparrow R^{d}} V_{\Lambda}(x \mid s \cap \tilde{\Lambda}) .
$$

Definition 1.4. For $n=1,2,3, \ldots$, let $d^{n} x$ be the projection of $n d$-dimensional Lebesque measure onto $X_{N}(\Lambda)$, so that $d^{n} x$ assigns total mass $|\Lambda|^{n}$ to $X_{N}(\Lambda)$, where $|\Lambda|$ is the Lebesque measure of $\Lambda$. The measure $d^{0} x$ assigns mass 1 to $X_{0}(\Lambda)$. Define the measure $v_{\Lambda}(d x)$ on $X(\Lambda)$ by

$$
v_{\Lambda}(d x)=\sum_{n=0}^{\infty} \frac{z^{n}}{n !} d^{n} x, \quad \text { where } z \text { is chemical activity. }
$$


Recall from Definition 1.1 that $s \in \Omega$ can be written as $s=s_{\Lambda} \vee \hat{s}$. For any $x \in X(\Lambda)$ we can form the configuration $x \vee \hat{s}$. This is just the configuration obtained from $s$ by changing the coordinates of $s$ in $\Lambda$ to $x$.

Definition 1.5. Corresponding to a potential $V$ and each $s \in R_{\Lambda}$, we define a measure $\mu_{\Lambda}(\cdot \mid s)$ on $X(\Lambda)$ by

$$
\mu_{\Lambda}(d x \mid s)=\frac{\exp \left(-\beta V_{\Lambda}(x \mid \hat{s})\right)}{Z_{\Lambda}(s)} v_{\Lambda}(d x),
$$

where $\beta$ is inverse temperature and $Z_{\Lambda}(s)$ makes $\mu_{\Lambda}(\cdot \mid s)$ a probability measure.

We note that $1 \leqq Z_{n}(s)<\infty$.

Definition 1.6. For a bounded Borel set $\Lambda \subset \mathbb{R}^{d}$ and a bounded $S$-measurable realvalued function $f$ on $\Omega$ define

We also let $\tau_{a}$ denote $\tau_{\Lambda_{a}}$.

$$
\tau_{\Lambda} f(s)= \begin{cases}\int_{X(\Lambda)} f(x \vee \hat{s}) \mu_{\Lambda}(d x \mid s) & \text { if } \quad s \in \mathbb{R}_{\Lambda} \\ 0 & \text { otherwise. }\end{cases}
$$

Definition 1.7. A probability measure $\sigma$ on $(\Omega, S)$ is a Gibbs state for the interaction $V$ if and only if

$$
\sigma\left(\tau_{\Lambda} f\right)=\sigma(f)
$$

for every $S$-measurable bounded function $f$ on $\Omega$, and every bounded Borel set $\Lambda \subset \mathbb{R}^{d}$.

We introduce next an $S$-measurable set $U \subset \Omega$, a vector space of functions $F(U)$ on $U$, and a linear map $T: F(U) \rightarrow F(U)$, defined in analogy with Gross [6].

Definition 1.8. Let $U=\bigcap_{i=1}^{\infty} R_{\Lambda_{i}}$, where $\Lambda_{i}$ is the cell defined by (1.1) and (1.2).

We point out that $\sigma(U)=1$ for any Gibbs state $\sigma$, since $1=\sigma(1)=\sigma\left(\tau_{A_{i}} 1\right)$ $=\sigma\left(R_{\Lambda_{i}}\right)$.

Definition 1.9. A function $f: U \rightarrow \mathbb{R}$ is a cylinder function on $U$ if there exists a bounded set $B \subset \mathbb{R}^{d}$ such that $f(s)=f(s \cap B)$ for all $s \in U$.

Definition 1.10. The space $F(U)$ is the completion in the infinity norm $\left(|\cdot|_{\infty}\right)$ of $\left\{f: U \rightarrow \mathbb{R} \mid f\right.$ is an $S$-measurable cylinder function and $\left.|f|_{\infty}<\infty\right\}$.

We use $S$ to denote both the $\sigma$-algebra on $\Omega$ and the induced $\sigma$-algebra in $U \subset \Omega$.

We point out that the operators $\tau_{\Lambda}$ as defined above act only on functions on $\Omega$. However, with a slight modification $\tau_{A}$ can be defined on $F(U)$. Thus to every $f \in F(U)$ we associate the function $g_{f}$ on $\Omega$ defined as follows,

$$
g_{f}(s)=\left\{\begin{array}{lll}
f(s) & \text { if } & s \in U \\
0 & \text { if } & s \in \Omega \backslash U .
\end{array}\right.
$$

The function $\tau_{\Lambda} g_{f}$ is well defined and for $f \in F(U)$, we define

$$
\tau_{\Lambda} f(s)=\tau_{\Lambda} g_{f}(s) \text { for } s \in U .
$$


Only those potentials $V$ for which $\tau_{\Lambda}: F(U) \rightarrow F(U)$ for every bounded Borel set $\Lambda \subset \mathbb{R}^{d}$ will be considered from this point on. Conditions guaranteeing this property will be given later.

To simplify notation, let $\tau_{i}=\tau_{\Lambda_{i}}, i=1,2,3, \ldots$ As in Gross $[6,7]$

$$
T_{n} f=\lim _{p \rightarrow \infty} \tau_{n} \tau_{n+1} \ldots \tau_{n+p} f
$$

exists for all $f \in F(U)$. We let $T=T_{1}$. Note that $\left|T_{n}\right|_{\infty}=1$ for all $n$.

In what follows we will have need of a metric $\varrho$ on $X\left(\Lambda_{a}\right)$ for each $a \in \mathbb{Z}^{d}$.

Let $s_{a}$ and $t_{a}$ be two non-empty configurations in $\Lambda_{a}$. Order the points in each configuration so that $s_{a}=\left(x^{1}, \ldots, x^{n}\right)$ and $t_{a}=\left(y^{1}, \ldots, y^{m}\right), x^{i}, y^{i} \in \Lambda_{a}$. Now define a metric $\varrho_{1}$ on $X\left(\Lambda_{a}\right)$ by

$$
\begin{gathered}
\varrho_{1}(\emptyset, \emptyset)=0, \\
\varrho_{1}(s, \emptyset)=\varrho(\emptyset, s)=n, \\
\varrho_{1}(s, t)=\varrho_{1}(t, s)= \begin{cases}\min _{p \in S_{n}} \sum_{i=1}^{n} d_{E}\left(x^{i}, y\right)^{q(i)}, & \text { if } m=n, \\
\min _{p \in S_{n}} \sum_{i=1}^{n} d_{E}\left(x^{i}, y^{p(i)}\right)+m-n & \text { if } \quad m \geqq n .\end{cases}
\end{gathered}
$$

Remark 1.1. The triangle inequality holds for dimensions $1,2,3$, and 4 with our present definition of $\varrho_{1}$. In the case of higher dimensions $d$ and/or cells $\Lambda_{a}$ with sides of length $\lambda \neq 1$, we can modify the definition of $\varrho_{1}$ to obtain a metric as follows. Replace " $n$ " in (1.14) by " $C n$ " and " $m-n$ " in (1.15) by " $C(m-n)$ ", where $C$ is any constant with $C \geqq \lambda \sqrt{d} / 2$.

Definition 1.11. Let $\varrho=\varrho_{1}+\varrho_{2}$, where $\varrho_{2}$ is the discrete metric on $X\left(\Lambda_{a}\right)$.

We now use the metric $\varrho$ to define norms on $F(U)$. For an $S$-measurable, real valued function $f$ on $U$ and $a \in \mathbb{Z}^{d}$, define

$$
\delta_{a}(f)=\sup \left\{\frac{|f(s)-f(t)|}{\varrho\left(s_{a}, t_{a}\right)}: s, t \in U, s_{b}=t_{b} \text { for all } b \in \mathbb{Z}^{d} \text { except when } b=a\right\} .
$$

Let $\mathscr{L}(U)$ denote the set of real $S$-measurable functions $f$ with $\delta_{a}(f)<\infty$ for each $a \in \mathbb{Z}^{d}$. This set $\mathscr{L}(U)$ is a linear space with semi-norms $\delta_{a}(f)$, the intersection of whose kernels, is the set of constant functions on $U$. The quotient space, $\mathscr{L}(U)$ modulo the constant functions, is a linear space with induced semi-norms which we also call $\delta_{a}(f), a \in \mathbb{Z}^{d}$.

Let $\mu_{a}$ be a real signed measure on $X\left(\Lambda_{a}\right)$ such that

If $f \in \mathscr{L}(U)$, then

$$
\int_{x\left(\Lambda_{a}\right)} \varrho(x, \emptyset)\left|\mu_{a}\right|(d x)<\infty .
$$

$$
\begin{aligned}
\int_{X\left(\Lambda_{a}\right)}|f(x \vee \hat{s})|\left|\mu_{a}\right|(d x) & \leqq \int_{X\left(\Lambda_{a}\right)}|f(x \vee \hat{s})-f(\emptyset)|\left|\mu_{a}\right|(d x)+|f(\emptyset)|\left\|\mu_{a}\right\|_{\mathrm{var}} \\
& \leqq \delta_{a}(f) \int_{X\left(\Lambda_{a}\right)} \varrho(x, \emptyset)\left|\mu_{a}\right|(d x)+|f(\emptyset)|\left\|\mu_{a}\right\|_{\mathrm{var}} \\
& <\infty
\end{aligned}
$$

where $\left\|\mu_{a}\right\|_{\text {var }}$ is the total variation of $\mu_{a}$. Thus $\int_{x\left(\Lambda_{a}\right)} f(x \vee \hat{s}) \mu_{a}(d x)$ is well defined. 
Let $\mathscr{L}_{a}(U)$ be the subspace of $\mathscr{L}(U)$ consisting of functions which depend only on configurations in $\Lambda_{a}$. If $\mu_{a}\left[X\left(\Lambda_{a}\right)\right]=0$, then $\mu_{a}$ is a linear functional on $\mathscr{L}_{a}(U)$ modulo the constant functions. We let $\left\|\mu_{a}\right\|^{a}$ be the dual space norm of $\mu_{a}$, that is,

$$
\left\|\mu_{a}\right\|^{a}=\sup _{\substack{\delta_{a}(f)=1 \\ f \in \mathscr{L}_{a}(U)}}\left|\mu_{a}(f)\right| .
$$

Observe that $\left\|\mu_{a}\right\|^{a}<\infty$ because

$$
\begin{aligned}
\left|\int_{X\left(\Lambda_{a}\right)} f(x) \mu_{a}(d x)\right| & \leqq\left|\int_{X\left(\Lambda_{a}\right)} f(x)-f(\emptyset) \mu_{a}(d x)\right|+\left|f(\emptyset) \int_{X\left(\Lambda_{a}\right)} \mu_{a}(d x)\right| \\
& \leqq \int_{X\left(\Lambda_{a}\right)}|f(x)-f(\emptyset)|\left|\mu_{a}\right|(d x)+0 \\
& \leqq \delta_{a}(f) \int_{X\left(\Lambda_{a}\right)} \varrho(x, \emptyset)\left|\mu_{a}\right|(d x)<\infty .
\end{aligned}
$$

We see from this computation that

$$
\left\|\mu_{a}\right\|^{a} \leqq \int_{X\left(\Lambda_{a}\right)} \varrho(x, \emptyset)\left|\mu_{a}\right|(d x)
$$

for a real signed measure $\mu_{a}$ on $X\left(\Lambda_{a}\right)$ with total mass zero.

Definition 1.12. For a potential $V$, define

$$
\begin{gathered}
\varrho_{a, b}=\sup \left\{\frac{\left\|\mu_{b}(\cdot \mid s)-\mu_{b}(\cdot \mid t)\right\|^{b}}{\varrho\left(s_{a}, t_{a}\right)}: s, t \in U \text { and } s=t \text { except at } a\right\}, \\
\text { where } \mu_{b}(\cdot \mid s)=\mu_{A_{b}}(\cdot \mid s) \text { is given by (2.3.2). }
\end{gathered}
$$

Let $d(\cdot, \cdot)$ be a semi-metric on $\mathbb{Z}^{d}$. We end this section with the following notation.

Definition 1.13.

$$
\begin{gathered}
\beta_{a, b}=\varrho_{a, b} e^{d(a, b)} \text { for } a \neq b, \text { and } \beta_{a, a}=0 \text { for all } a, b \in \mathbb{Z}^{d} . \\
\alpha=\sup _{b \in \mathbb{Z}^{d}} \sum_{a \in \mathbb{Z}^{d}} \beta_{a, b}, \\
\|f\|_{a}=\sum_{b \in \mathbb{Z}^{d}} e^{d(a, b)} \delta_{a}(f), \text { for } f \in \mathscr{L}(U), \\
L(U)=\left\{f \in F(U):\|f\|_{a}<\infty \text { for every } a \in \mathbb{Z}^{d}\right\} .
\end{gathered}
$$

Remark 1.2. With the translation invariance of the potential $V$, it is easy to check that $\varrho_{a, b}=\varrho_{b, a}$. Hence we also have $\beta_{a, b}=\beta_{b, a}$ and $\alpha=\sup _{a \in \mathbb{Z}^{d}} \sum_{b \in \mathbb{Z}^{d}} \beta_{a, b}$.

\section{Section 2. Theorems on Decay}

In this section we outline the continuum extension of Gross' Theorems 1 and 2 in [6].

With the definitions just given, it is easily checked that

$$
\delta_{a}\left(\tau_{b} f\right) \leqq \delta_{a}(f)+\varrho_{a, b} \delta_{b}(f)
$$


holds for $f \in F(U)$. From this Gross' Lemma 3.1 in [6] and his Corollary 3.2 can be expressed, for the continuum case as

Corollary 2.1. If $\alpha<1$, then for all $f \in L(U)$ and all $a \in \mathbb{Z}^{d}$, $\|T f\|_{a} \leqq \alpha\|f\|_{a}$.

The proof of Corollary 3.3 in [6] does not hold under the present circumstances. An alternative proof adapted from unpublished work of Gross is given here.

Lemma 2.1. For every $g \in F(U)$ and every $s, t \in U$,

$$
|g(s)-g(t)| \leqq \sum_{k=1}^{\infty} \delta_{k}(g) \varrho\left(s_{k}, t_{k}\right) .
$$

Proof. For every $\varepsilon \geqq 0$ there exists a cylinder function $g_{N}$ such that $\left|g_{N}-g\right|_{\infty}<\varepsilon$ and such that $g_{N}(s)$ depends only on the coordinates of $s$ in the cubes $\Lambda_{1}, \ldots, \Lambda_{N}$, for every $s \in U$. Define $\Lambda=\bigcup_{k=1}^{N} \Lambda_{k}$ and $s^{\prime}=s_{\Lambda} \vee t_{\mathbb{R}^{d} / \Lambda}$. Then

$$
g_{N}\left(s^{\prime}\right)=g_{N}(s) \text { so that }\left|g(s)-g\left(s^{\prime}\right)\right| \leqq\left|g(s)-g_{N}(s)\right|+\left|g_{N}\left(s^{\prime}\right)-g\left(s^{\prime}\right)\right| \text {, }
$$

and hence $\left|g(s)-g\left(s^{\prime}\right)\right| \leqq 2 \varepsilon$. Thus

$$
\begin{aligned}
|g(s)-g(t)| & \leqq\left|g(s)-g\left(s^{\prime}\right)\right|+\left|g\left(s^{\prime}\right)-g(t)\right| \\
& \leqq 2 \varepsilon+\sum_{k=1}^{N} \delta_{k}(g) \varrho\left(s_{k}^{\prime}, t_{k}\right),
\end{aligned}
$$

where the last inequality follows from the triangle inequality and the definition of $\delta_{k}(g)$. But now since $s_{k}^{\prime}=s_{k}$ for $k=1, \ldots, N$ we can write

$$
\begin{aligned}
|g(s)-g(t)| & \leqq 2 \varepsilon+\sum_{k=1}^{N} \delta_{k}(g) \varrho\left(s_{k}, t_{k}\right) \\
& \leqq 2 \varepsilon+\sum_{k=1}^{\infty} \delta_{k}(g) \varrho\left(s_{k}, t_{k}\right) .
\end{aligned}
$$

Since this inequality holds for all $\varepsilon>0$, we get

$$
|g(s)-g(t)| \leqq \sum_{k=1}^{\infty} \delta_{k}(g) \varrho\left(s_{k}, t_{k}\right)
$$

This completes the proof.

Theorem 2.1. For a given potential $V$, suppose that $\alpha<1$. Then there is at most one probability measure $\sigma$ on $(\Omega, S)$ such that:

$$
\begin{gathered}
\sigma\left[\varrho\left(s_{k}, \emptyset\right)\right] \leqq M \text { for some positive real number } M \\
\text { and all integers } k \geqq 1, \\
\sigma\left(\tau_{k} f\right)=\sigma(f) \text { for all bounded } S \text {-measurable functions } f \\
\text { on } \Omega \text { and all integers } k \geqq 1 .
\end{gathered}
$$

Furthermore, if such a probability measure $\sigma$ exists, then

$$
\lim _{n \rightarrow \infty}\left(T^{n} f\right)(\emptyset)=\sigma(f) .
$$


Proof. The semi-metric $d(\cdot, \cdot)$ plays no role in the proof of this theorem, since if $\alpha<1$ for any semi-metric $d(\cdot, \cdot)$ not identically zero, then $\alpha<1$ for the semi-metric identically equal to zero. Without loss of generality, then, we will take $d(\cdot, \cdot) \equiv 0$ in this proof. In this case $\|f\|_{a}$ has no dependence on $a \in \mathbb{Z}^{d}$ and we will write $\|f\|=\sum_{b \in \mathbb{Z}^{d}} \delta_{b}(f)$. From Corollary 2.1 we have,

$$
\lim _{n \rightarrow \infty}\left\|T^{n} f\right\|=0, \text { for } f \in L(U) .
$$

Now let $\sigma$ be a probability measure on $\Omega$ with the property (2.3). Then $\sigma\left(\tau_{k} f\right)=\sigma(f)$ for $f \in L(U) \subset F(U)$. Since $T f$ is well defined in this case, it follows that $\sigma(T f)=\sigma(f)$ and $\sigma\left(T^{n} f\right)=\sigma(f)$ for $n=1,2,3, \ldots$ We can therefore write

$$
\sigma(f)=\sigma\left(T^{n} f\right)=\sigma\left[T^{n} f-\left(T^{n} f\right)(\emptyset)\right]+\left(T^{n} f\right)(\emptyset) .
$$

By Lemma 2.1

$$
\sigma\left[T^{n} f-\left(T^{n} f\right)(\emptyset)\right] \leqq \sigma\left[\sum_{k=1}^{\infty} \delta_{k}\left(T^{n} f\right) \varrho\left(s_{k}, \emptyset\right)\right]
$$

By (2.2) we can write

$$
\sigma\left[T^{n} f-\left(T^{n} f\right)(\emptyset)\right] \leqq M\left\|T^{n} f\right\| .
$$

It follows from (2.5) and (2.6) that $\lim _{n \rightarrow \infty}\left(T^{n} f\right)(\emptyset)$ exists and equals $\sigma(f)$ for any $f \in L(U)$. Now $L(U)$ contains all bounded cylinder functions on $U$. Since $\sigma(\Omega \backslash U)=0$, a standard argument from measure theory shows that $\sigma$ is uniquely determined on $(\Omega, S)$. This completes the proof.

We now state the following modified lemma and theorems in analogy to [6].

Lemma 2.2. If $\int_{X\left(\Lambda_{k}\right)}\left[\int_{X\left(\Lambda_{k}\right)} \varrho(x, y) \mu_{k}(d y \mid s)\right]^{2} \mu_{k}(d y \mid s) \leqq C$ for some real number $C$ and every $s \in U$ and every integer $k \geqq 1$, then

$$
|T(f g)-T(f) T(g)|_{\infty} \leqq C \sum_{n=1}^{\infty} \delta_{n}\left(T_{n+1} f\right) \delta_{n}\left(T_{n+1} g\right)
$$

for all $f, g \in L(U)$.

Theorem 2.2. Let $\sigma$ be a probability measure on $\Omega$ such that $\sigma\left(\tau_{k} f\right)=\sigma(f)$ for every bounded $S$-measurable function $f$ on $\Omega$ and every integer $k \geqq 1$. Assume that the following conditions hold:

$$
\int_{X\left(\Lambda_{k}\right)}\left[\int_{x\left(\Lambda_{k}\right)} \varrho(x, y) \mu_{k}(d y \mid s)\right]^{2} \mu_{k}(d x \mid s) \leqq C
$$

for some real number $C$, all $k \geqq 1$, and all $s \in U$,

$\sigma\left[\varrho\left(s_{k}, \emptyset\right)\right] \leqq M$ for some real number $M$ and all $k \geqq 1$,

$$
\alpha<1 \text {. }
$$

Then $\sigma$ is unique and

$$
\begin{gathered}
|\sigma(f g)-\sigma(f) \sigma(f)| \leqq C e^{-d(a, b)}\|f\|_{a}\|g\|_{b}(1-\alpha)^{-2}\left(1-\alpha^{2}\right)^{-1} \\
\text { for all } a, b \in \mathbb{Z}^{d} \text { and } f, g \in L(U)
\end{gathered}
$$


Definition 2.1. Let $g$ be a real valued function on $U$. We define $g_{a}(s)=g\left(s^{(a)}\right)$, where $s_{b}^{(a)}=s_{b+a}$, and $a, b \in \mathbb{Z}^{d}$.

Theorem 2.3. Assume that the semi-metric $d(\cdot, \cdot)$ is translation invariant on $\mathbb{Z}^{d}$. If $\alpha(\alpha+1)<1$, with the same conditions as in Theorem 2.2, then for any functions $f, g \in L(U)$ and any $c \in \mathbb{Z}^{d}$,

$$
\sum_{a \in \mathbb{Z}^{d}}\left|\sigma\left(f g_{a}\right)-\sigma(f) \sigma\left(g_{a}\right)\right| e^{d(a, c)} \leqq C\|f\|_{c}\|g\|_{0}(1-\alpha)^{-1}\left(1-\alpha-\alpha^{2}\right)^{-1}
$$

where the subscript 0 on $\|g\|_{0}$ refers to the origin in $\mathbb{Z}^{d}$.

The proofs of Lemma 2.2, Theorem 2.2 and Theorem 2.3 are similar to the proofs of the corresponding results in [6] with straightforward modifications which follow from the definitions given in the previous section.

\section{Section 3. Bounds}

In this section we describe a class of potentials whose Gibbs states satisfy the hypotheses of the theorems of the preceding section in the case of high temperature or low activity.

Much of what follows is based upon inequality (1.18), which for a given potential $V$ can be expressed as

$$
\left\|\mu_{b}(\mid s)-\mu_{b}(\mid t)\right\|^{b} \leqq \int_{X\left(\Lambda_{b}\right)} \varrho(x, \emptyset)\left|\frac{\exp \left(-\beta V_{b}(x \mid \hat{s})\right)}{Z_{b}(s)}-\frac{\exp \left(-\beta V_{b}(x \mid \hat{t})\right)}{Z_{b}(t)}\right| v_{b}(d x)
$$

where we have abbreviated and will continue to abreviate $V_{b}(x \mid \hat{s})$ for $V_{A_{b}}(x \mid \hat{s}), v_{b}$ for $v_{\Lambda_{b}}$, and $Z_{b}(s)$ for $Z_{\Lambda_{b}}$.

We begin by listing two conditions to which we will refer several times later.

Condition 3.1. There exist $r_{0} \geqq 0$, a positive decreasing function $\psi(r)$ on $\left(r_{0}, \infty\right)$, and constants $K_{N}$ which depend on $N$, such that:

a) $\sup _{b} \sum_{a \in A} e^{d(a, b)} \psi\left[d_{E}\left(\Lambda_{a}, \Lambda_{b}\right)\right]<\infty$, where $A=\left\{a \in \mathbb{Z}^{d}: d_{E}\left(\Lambda_{b}, \Lambda_{a}\right)>r_{0}\right\}$,

b) $\phi_{N}\left(x^{1}, \ldots, x^{N}\right) \mid \leqq K_{N} \psi(r)$ for $r=\max _{1 \leqq i, j \leqq N}\left[d_{E}\left(x^{i}, x^{j}\right)\right]>r_{0}$,

c) $\mid\left(\phi_{N}\left(x^{1}, \ldots, x^{N}\right)-\phi_{N}\left(y^{1}, \ldots, y^{N}\right) \mid<K_{N} \psi(r) \varrho_{1}(x, y)\right.$, for

$$
\begin{aligned}
& r=\min \left\{\max _{i, j}\left[d_{E}\left(x^{i}, x^{j}\right)\right], \max _{i, j}\left[d_{E}\left(y^{i}, y^{j}\right)\right]\right\}>r_{0}, \text { where } \\
& x=\left(x^{1}, \ldots, x^{N}\right), \quad y=\left(y^{1}, \ldots, y^{N}\right)
\end{aligned}
$$

Before stating Condition 3.2 we make the following definition. 
Definition 3.1.

$$
d \mu_{\lambda, b}^{s, t}=\frac{\exp \left(-\beta\left[\lambda V_{b}(x \mid \hat{s})+(1-\lambda) V_{b}(x \mid \hat{t})\right]\right)}{Z_{b}(\lambda, s, t)} v_{b}(d x)
$$

and

$$
Z_{b}(\lambda, s, t)=\int_{x\left(\Lambda_{b}\right)} \exp \left(-\beta\left[\lambda V_{b}(x \mid \hat{s})+(1-\lambda) V_{b}(x \mid \hat{t})\right]\right) v_{b}(d x) .
$$

When $s, t \in U$ and $b \in \mathbb{Z}^{d}$ are fixed, we will write

$$
d \mu_{\lambda}=d \mu_{\lambda, b}^{s, t} .
$$

Condition 3.2. For $k>0$ given, there exists a function $M(z, \beta)$ decreasing in each argument $z$ and $\beta$ such that

$$
\int_{X\left(\Lambda_{b}\right)}|x|^{k} d \mu_{\lambda, b}^{s, t} \leqq z M(z, \beta) \quad \text { for all } 0 \leqq \lambda \leqq 1
$$

$a, b \in \mathbb{Z}^{d}$, and $s, t \in U$ which differ only on $\Lambda_{a} \neq \Lambda_{b}$.

Remark 3.1. It is easy to show that if there exists a $B \geqq 0$ such that $V_{b}(x \mid \hat{s}) \geqq-B|x|$ for every $s \in U, x \in X\left(\Lambda_{b}\right)$, and $b \in \mathbb{Z}^{d}$, then Condition 3.2 is satisfied, for $k=2$ with $M(z, \beta)=z e^{2 \beta B}+e^{\beta B}$. Thus, any positive potential satisfies Condition 3.2 (with $k=2$ ).

Theorem 3.1. Suppose that a potential V satisfies part a) and b) of Condiction 3.1 and the following three conditions:

$$
\begin{gathered}
\phi_{N_{0}} \text { is hard core for some } N_{0} \geqq 2, \\
\phi_{N}\left(x^{1}, \ldots, x^{N}\right)=0 \text { whenever } \max _{i j} d_{E}\left(x^{i}, x^{j}\right)>R_{0} \\
\text { for some given } R_{0}>0, \text { for } N \geqq 3, \\
\inf \left\{\phi_{N}(y): y \in \Omega_{F},|y|=N, N \geqq 2\right\}>-\infty .
\end{gathered}
$$

Then $V$ satisfies Condition 3.2, with $k=2$.

Proof. We show that the hypothesis of Remark 3.1 is satisfied. Let $C_{n}$ denote the cube of side $2 n$ whose center is $b \in \mathbb{Z}^{d}$. By (3.3) there exists a positive integer $n$ such that

$$
V_{b}(x \mid \hat{s})=V_{b}\left(x \mid \hat{s} \cap C_{n}\right)+\sum_{\Lambda_{k} \subset C_{n}^{c}} \sum_{\substack{x^{i} \in x \\ s^{j} \in \lambda_{k}}} \phi_{2}\left(x^{i}, s^{j}\right),
$$

where $C_{n}^{c}$ denotes the complement in $\mathbb{R}^{d}$ of $C_{n}$, and the sum $\sum_{A_{k} \subset C_{n}^{c}}$ is taken in 
lexicographic order with respect to $k \in \mathbb{Z}^{d}$. We can write

$$
V_{b}\left(x \mid \hat{s} \cap C_{n}\right)=V(x)+\sum_{N=2}^{|x|+\left|\hat{s} \cap C_{n}\right|} \sum_{\substack{y \subset x \vee \hat{s} \\ y \subset C_{n} \\ y \mid=N \\ y \cap s \cap x \neq \emptyset}} \phi_{N}(y)
$$

for $x \in X\left(\Lambda_{b}\right)$. Recall from Sect. 1 that we are assuming that $V$ is stable. Thus

$$
V(x) \geqq-B_{1}|x|
$$

for some $B_{1} \geqq 0$ and all $x \in \Omega_{F}$. By the definition of $U$ and since $\phi_{N_{0}}$ is hard core, $\left|\hat{s} \cap C_{n}\right|$ is bounded for all $s \in U$. It follows that there exists an integer $K>0$ such that if the double sum, DS, on the right side of (3.6) is finite, then $\left|x \vee\left(s \cap C_{n}\right)\right|<K$. Thus by (3.4) there exists $B_{2}>0$ such that $\mathrm{DS}>-B_{2}$ for all $s \in U$ and $x \in X\left(\Lambda_{b}\right)$. Combining this with (3.6) and (3.7) gives

$$
V_{b}\left(x \mid \hat{s} \cap C_{n}\right) \geqq-B_{1}|x|-B_{2} .
$$

Now by (3.2) and the definition of $U$, there exists $M>0$ such that $\left|s \cap \Lambda_{k}\right|<M$ for all $k \geqq 1$ and $s \in U$. Thus going back to (3.5), we can write

$$
\left.\sum_{\Lambda_{k} \subset C_{n}^{c}} \sum_{\substack{x^{i} \in X \\ S^{j} \in \Lambda_{k}}} \phi_{2}\left(x^{i}, S^{j}\right) \geqq-|x| M \sum_{\Lambda_{k} \subset C_{n}^{c}} K_{2} \psi\left[\Lambda_{b}, \Lambda_{k}\right)\right],
$$

by parts a) and b) of Condition 3.1. Combining this with (3.8) and (3.5) gives

$$
V_{b}(x \mid \hat{s}) \geqq-B_{3}|x|-B_{1}
$$

for some $B_{3}>0$. Hence there exists a $B>0$ such that $V_{b}(x \mid \hat{s}) \geqq-B|x|$ for all $s \in U$ and $x \in X\left(\Lambda_{b}\right)$. The conclusion now follows from Remark 3.1. This completes the proof.

The next two lemmas deal with the hypotheses (2.7) and (2.8) for Theorems 2.2 and 2.3.

Lemma 3.1. Let $V$ satisfy Condition 3.2. with $k=1$ and let $\sigma$ be a Gibbs state for $V$. Then

$$
\sigma\left[\varrho\left(s_{k}, \emptyset\right)\right] \leqq 2 z M(z, \beta)
$$

for all $s \in U$ and $k \geqq 1$. Hence if $z$ and $\beta$ are bounded above by some constant $K>0$, then there exists a positive number $M$ such that for all $k \geqq 1$,

$$
\sigma\left[\varrho\left(s_{k}, \emptyset\right)\right] \leqq M .
$$

Proof. By Condition 3.2 and since $\varrho(x, \emptyset) \leqq 2|x|$,

$$
\begin{aligned}
\tau_{k} \varrho(x, \emptyset)(s) & \leqq \int_{X\left(\Lambda_{k}\right)} 2|x| \frac{\exp \left(-\beta V_{a_{k}}(x \mid \hat{s})\right)}{Z_{a_{k}}(s)} v_{a_{k}}(d x) \\
& \leqq 2 z M(z, \beta) .
\end{aligned}
$$

Thus,

$$
\sigma\left[\tau_{k} \varrho(x, \emptyset)(s)\right] \leqq 2 z M(z, \beta)
$$


Since $\sigma$ is a Gibbs state, we have $\sigma\left(\tau_{k} f\right)=\sigma(f)$ for all bounded $S$-measurable functions $f$. A straightforward application of the monotone convergence theorem shows that

$$
\sigma\left[\varrho\left(s_{k}, \emptyset\right)\right]=\sigma\left[\tau_{k} \varrho(x, \emptyset)(s)\right] .
$$

Now combining this with (3.9) gives the desired conclusion. This completes the proof.

Lemma 3.2. Let $K>0$ be given and suppose that $V$ satisfies Condition 3.2 with $k=1$, and $\sigma$ is a Gibbs state for $V$. If $z, \beta<K$, then there exists a constant $C$ such that for all $k \geqq 1$ and $s \in U$,

$$
\int_{X\left(\Lambda_{k}\right)}\left[\int_{X\left(\Lambda_{k}\right)} \varrho(x, y) \mu_{k}(d y \mid s)\right]^{2} \mu_{k}(d x \mid s) \leqq C .
$$

Proof. By the triangle inequality for the metric $\varrho(\cdot, \cdot)$,

$$
\varrho(x, y) \leqq \varrho(x, \emptyset)+\varrho(y, \emptyset) \leqq 2|x|+2|y| .
$$

Hence

$$
\begin{aligned}
\int_{x\left(\Lambda_{k}\right)} \varrho(x, y) \mu_{k}(d y \mid s) & \leqq 2|x|+\int_{x\left(\Lambda_{k}\right)} 2|y| \frac{\exp \left(-\beta V_{a_{k}}(y \mid \hat{s})\right)}{Z_{a_{k}}(s)} v_{a_{k}}(d y) \\
& \leqq 2|x|+2 z M(z, \beta)
\end{aligned}
$$

by Condition 3.2. Thus $\left[\int_{X\left(\Lambda_{k}\right)} \varrho(x, y) \mu_{k}(d x \mid s)\right]^{2}$ is bounded by a quadratic polynomial in $|x|$ whose coefficients are decreasing functions of $z$ and $\beta$. Since $z, \beta<K$, a second application Condition 3.2 now yields (3.10) for some constant $C$. This completes the proof.

In Sect. 1 we restricted our attention to those potentials for which $\tau_{\Lambda}: F(U) \rightarrow F(U)$, for every bounded Borel set $\Lambda \subset \mathbb{R}^{d}$. We now give conditions on the potential $V$ so that this is true.

Theorem 3.2. If $V$ has finite range, then $\tau_{\Lambda}: F(U) \rightarrow F(U)$ for every bounded Borel set $\Lambda \subset \mathbb{R}^{d}$.

Proof. Let $f \in F(U)$ and let $\left\{f_{n}\right\}$ be a sequence of cylinder functions in $F(U)$ converging uniformly to $f$. Let $C_{m}$ be the hypercube of side $2 m$ centered at the origin in $\mathbb{R}^{d}$. We will construct a sequence $\left\{g_{n}\right\}$ of cylinder functions in $F(U)$ converging to $\tau_{A} f$, thus showing that $\tau_{A} f \in F(U)$. Define

$$
g_{n}(s)=\int_{X(\Lambda)} f_{n}(x \vee \hat{s}) \frac{\exp \left(-\beta V_{\Lambda}\left(x \mid \hat{s} \cap C_{n}\right)\right)}{Z_{\Lambda}\left(s \cap C_{n}\right)} v_{\Lambda}(d x)
$$

and

$$
g_{n}^{\prime}(s)=\int_{X(\Lambda)} f(x \vee \hat{s}) \frac{\exp \left(-\beta V_{\Lambda}\left(x \mid \hat{s} \cap C_{n}\right)\right)}{Z_{\Lambda}\left(s \cap C_{n}\right)} v_{\Lambda}(d x) .
$$

Clearly $g_{n}$ is an $S$-measurable cylinder function in $F(U)$. By the triangle inequality, $\left|\tau_{A} f-g_{n}\right|_{\infty} \leqq\left|\tau_{\Lambda} f-g_{n}^{\prime}\right|_{\infty}+\left|g_{n}^{\prime}-g_{n}\right|_{\infty}$

$$
\leqq|f|_{\infty} \sup _{s \in U} \int_{X(\Lambda)}\left|\frac{\exp \left(-\beta V_{\Lambda}(x \mid \hat{s})\right)}{Z_{\Lambda}(s)}-\frac{\exp \left(-\beta V_{\Lambda}\left(x \mid \hat{s} \cap C_{n}\right)\right)}{Z_{\Lambda}\left(s \cap C_{n}\right)}\right| v_{\Lambda}(d x)+\left|f-f_{n}\right|_{\infty} .
$$


But since $V$ has finite range, there exists a positive integer $n_{0}$ such that for $n \geqq n_{0}$,

$$
V_{\Lambda}(x \mid \hat{s})=V_{\Lambda}\left(x \mid \hat{s} \cap C_{n}\right) \quad \text { for all } \quad x \in X(\Lambda)
$$

and $s \in U$. Thus for $n \geqq n_{0}$ (3.11) becomes

$$
\left|\tau_{\Lambda} f-g_{n}\right|_{\infty} \leqq\left|f-f_{n}\right|_{\infty},
$$

and since $f_{n} \rightarrow f$, we have that $g_{n} \rightarrow \tau_{\Lambda} f$. This completes the proof.

The proof of the following lemma is based on the proof of the lemma given in Simon [16].

Lemma 3.3. Let $\Lambda \subset \mathbb{R}^{d}$ be any bounded Borel set and let $f \in F(U)$. If $s, t \in U$ and

$$
\left\{x \in X(\Lambda): V_{\Lambda}(x \mid \hat{s})<\infty\right\}=\left\{x \in X(\Lambda): V_{\Lambda}(x \mid \hat{t})<\infty\right\}
$$

then

$$
\begin{gathered}
\int_{x(\Lambda)} f(x \vee \hat{s})\left|\frac{\exp \left(-\beta V_{\Lambda}(x \mid \hat{s})\right)}{Z_{\Lambda}(s)}-\frac{\exp \left(-\beta V_{\Lambda}(x \mid \hat{t})\right)}{Z_{\Lambda}(t)}\right| v_{\Lambda}(d x) \\
\leqq|f|_{\infty} \beta \sup \left\{\left|V_{\Lambda}(x \mid \hat{s})-V_{\Lambda}(x \mid \hat{t})\right|: x \in X(\Lambda) \cap U\right\} .
\end{gathered}
$$

Theorem 3.3. Let $V$ satisfy the following conditions :

a) $\phi_{N}\left(x^{1}, \ldots, x^{N}\right)=0$ whenever $\max _{i j} d_{E}\left(x^{i}, x^{j}\right)>R_{0}$, for some given $R$ and all $N \geqq 3$.

b) $V$ satisfies parts a) and b) of Condition 3.1,

c) $\phi_{N_{0}}$ is hard-core for some $N_{0} \geqq 2$.

Then $\tau_{\Lambda}: F(U) \rightarrow F(U)$ for all bounded Borel sets $\Lambda \subset \mathbb{R}^{d}$.

Proof. The proof is identical to the proof of Theorem 3.2 up to inequality (3.11). We proceed from there.

By the definition of $R_{A}^{0}$ we can choose $n$ so large that

$$
\left\{x \in X(\Lambda): V_{\Lambda}\left(x \mid \hat{s} \cap C_{n}\right)<\infty\right\}=\left\{x \in X(\Lambda): V_{\Lambda}(x \mid \hat{s})<\infty\right\} .
$$

We can thus apply Lemma 3.3 to get

$$
\left|\tau_{\Lambda} f-g_{n}\right|_{\infty} \leqq \beta|f|_{\infty} \sup \left\{\left|V_{\Lambda}\left(x \mid \hat{s} \cap C_{n}\right)-V_{\Lambda}(x \mid \hat{s})\right|: x \in X(\Lambda) \cap U, s \in U\right\}+\left|f-f_{n}\right|_{\infty} .
$$

Now for $n$ sufficiently large, we can write

$$
\left|V_{\Lambda}(x \mid \hat{s})-V_{\Lambda}\left(x \mid \hat{s} \cap C_{n}\right)\right|=\sum_{\Lambda_{k} \subset C_{n}^{c}} \sum_{x^{i} \in x} \sum_{s^{j} \in S \cap \Lambda_{k}} \phi_{2}\left(x^{i}, s_{j}\right)
$$

where $\Lambda_{k}$, as usual, refers to the cells with side of length one partioning $\mathbb{R}^{d}$, and $C_{n}^{c}$ denotes the complement in $\mathbb{R}^{d}$ of $C_{n}$. By Condition 3.1, for $n$ sufficiently large,

$$
\left|V_{\Lambda}(x \mid \hat{s})-V_{\Lambda}\left(x \mid \hat{s} \cap C_{n}\right)\right| \leqq \sum_{\Lambda_{k} \subset C_{n}^{c}} \sum_{x^{i} \in x} \sum_{s^{j} \in S \cap \Lambda_{k}} K_{2} \psi\left[d_{E}\left(\Lambda, \Lambda_{k}\right)\right]
$$


Now by the definition of $U$, and since $\phi_{N_{0}}$ is hard-core, there is an upper bound on the cardinality of $x \subset \Lambda$ and $s \cap \Lambda_{k} \subset \Lambda_{k}$ because $\Lambda$ and $\Lambda_{k}$ are bounded sets. Thus there is some constant $K>0$ such that

$$
\left|V_{\Lambda}(x \mid \hat{s})-V_{\Lambda}\left(x \mid \hat{s} \cap C_{n}\right)\right| \leqq K \sum_{\Lambda_{k} \subset C_{n}^{c}} \psi\left[d_{E}\left(\Lambda, \Lambda_{k}\right)\right] .
$$

But by Condition 3.1 and the boundedness of $\Lambda, \sum_{\Lambda_{k} \subset C_{n}^{c}} \psi\left[d_{E}\left(\Lambda, \Lambda_{k}\right)\right]<\infty$ and approaches zero as $n$ goes to infinity, independent of $x$ and $s$ in $U$. Hence by (3.13) and since $\left|f-f_{n}\right|_{\infty}$ converges to zero as $n$ approaches infinity, we have

$$
\left|\tau_{A} f-g_{n}\right|_{\infty} \rightarrow 0 \text { as } n \rightarrow 0,
$$

and hence $\tau_{\Lambda} g \in F(U)$. This completes the proof.

The remaining theorems in this section give bounds on $\alpha$.

We state without proof the following easy lemma.

Lemma 3.4. Let $V$ satisfy Condition 3.2 with $k=1$ or 2 . If $\Lambda_{a}$ and $\Lambda_{b}$ are distinct, and $s$ and $t$ are any two configurations in $U$ which differ only on $\Lambda_{a}$, then

$$
\int_{X\left(A_{b}\right)} \varrho(x, \emptyset)\left|\frac{\exp \left(-\beta V_{b}(x \mid \hat{s})\right)}{Z_{b}(s)}-\frac{\exp \left(-\beta V_{b}(x \mid \hat{t})\right)}{Z_{b}(t)}\right| v_{b}(d x) \leqq 4 z M(z, \beta) .
$$

The proof of the next lemma is similar to the proof of Lemma 3.3.

Lemma 3.5. Let $s$ and $t$ be any two configurations in $U$. If $\left\{x \in X\left(\Lambda_{b}\right): V_{b}(x \mid \hat{s})<\infty\right\}$ $=\left\{x \in X\left(\Lambda_{b}\right): V_{b}(x \mid \hat{t})<\infty\right\}$, then

$$
\begin{aligned}
& \int_{X\left(\Lambda_{b}\right)} \varrho(x, \emptyset)\left|\frac{\exp \left(-\beta V_{b}(x \mid \hat{s})\right)}{Z_{b}(s)}-\frac{\exp \left(-\beta V_{b}(x \mid \hat{t})\right)}{Z_{b}(t)}\right| v_{b}(d x) \\
& \quad \leqq 2 \beta \int_{0}^{1} \int_{X\left(\Lambda_{b}\right)} \varrho(x, \emptyset)\left|V_{b}(x \mid \hat{s})-V_{b}(x \mid \hat{t})\right| d \mu_{\lambda} d \lambda .
\end{aligned}
$$

In the case that $V$ is a pair potential, there exists an even function $\phi: \mathbb{R}^{d} \rightarrow(-\infty,+\infty]$ such that $V(x)=\sum_{i<j} \phi\left(x^{i}-x^{j}\right)$ for $x \in \Omega_{F}$. We use this notation in the following lemma.

Lemma 3.6. If $V$ is a pair potential which satisfies Conditions 3.1 and 3.2 with $k=2$, and if $d_{E}\left(\Lambda_{a}, \Lambda_{b}\right)=r>r_{0}$ and $s$ and $t$ are any two configurations in $U$ which differ only on $\Lambda_{a}$, then

$$
\varrho_{a, b} \leqq 4 \beta z M(z, \beta) \psi(r) .
$$

Proof. Let $s_{a}$ and $t_{a}$ have coordinates in $\mathbb{R}^{d}$ given by $s_{a}=\left(s^{1}, s^{2}, \ldots, s^{n}\right)$ and $t_{a}=\left(t^{1} t^{2}, \ldots, t^{m}\right)$. Assume that $m \geqq n$ and that the coordinates of $s_{a}$ and $t_{a}$ are ordered so that

$$
\sum_{i=1}^{n} d_{E}\left(s^{i}, t^{i}\right)+m-n=\varrho_{1}\left(s_{a}, t_{a}\right)
$$


Let the coordinates of $x \in X\left(\Lambda_{b}\right)$ be given by $x=\left(x^{1}, x^{2}, \ldots, x^{k}\right)$. By the definitions of $V_{b}(x \mid \hat{s})$ and $V_{b}(x \mid \hat{t})$ and since $s=t$ except on $\Lambda_{a}$, we have

$$
\begin{aligned}
\left|V_{b}(x \mid \hat{s})-V_{b}(x \mid \hat{t})\right| \leqq & \sum_{i=1}^{k} \sum_{j=1}^{n}\left|\phi\left(x^{i}-s^{j}\right)-\phi\left(x^{i}-t^{j}\right)\right| \\
& +\sum_{i=1}^{k} \sum_{j=n+1}^{m}\left|\phi\left(x^{i}-t^{j}\right)\right| \\
\leqq & k \psi(r) \sum_{j=1}^{n} d_{E}\left(s^{j}, t^{j}\right)+k \psi(r)(m-n),
\end{aligned}
$$

where in the third line, we have used Condition 3.1. By (3.17) we can write

$$
\left|V_{b}(x \mid \hat{S})-V_{b}(x \mid \hat{t})\right| \leqq|x| \psi(r) \varrho_{1}\left(s_{a}, t_{a}\right)
$$

Now combining (3.19) with (3.15) we get

$$
\begin{aligned}
& \int_{X\left(\Lambda_{b}\right)} \varrho(x, \emptyset)\left|\frac{\exp \left(-\beta V_{b}(x \mid \hat{s})\right)}{Z_{b}(s)}-\frac{\exp \left(-\beta V_{b}(x \mid \hat{t})\right)}{Z_{b}(t)}\right| v_{b}(d x) \\
& \leqq 2 \beta \psi(r) \varrho_{1}\left(s_{a}, t_{a}\right) \int_{0}^{1} \int_{x\left(\Lambda_{b}\right)} \varrho(x, \emptyset)|x| d \mu_{\lambda} d \lambda .
\end{aligned}
$$

Since $\varrho(x, \emptyset)=|x|+1$ if $x \neq \emptyset, \varrho(x, \emptyset)|x|=|x|^{2}+|x| \leqq 2|x|^{2}$. Thus by Condition 3.2, with $k=2$

$$
\int_{X\left(\Lambda_{b}\right)} \varrho(x, \emptyset)\left|\frac{\exp \left(-\beta V_{b}(x \mid \hat{s})\right)}{Z_{b}(s)}-\frac{\exp \left(-\beta V_{b}(x \mid \hat{t})\right)}{Z_{b}(t)}\right| v_{b}(d x) \leqq 4 \beta z M(z, \beta) \varrho_{1}\left(s_{a}, t_{a}\right) \psi(r),
$$

and combining this with (3.1) gives us

$$
\varrho_{a, b} \leqq 4 \beta z M(z, \beta) \psi(r) .
$$

This completes the proof.

Theorem 3.4. Let $K>0$ and $\eta>0$ be given. If $V$ is a pair potential and satisfies Conditions 3.1 and 3.2 with $k=2$, and if $z, \beta<K$, then there exists $C_{k}>0$ such that if $z<C_{k}$, then $\alpha<\eta$ for $\beta$ or $z$ sufficiently small.

Proof. From the definition of $\alpha$ it follows that

$$
\alpha \leqq \sup _{b} \sum_{a \in A} e^{d(a, b)} \varrho_{a, b}+\sup _{b} \sum_{a \in A^{c}} e^{d(a, b)} \varrho_{a, b},
$$

where $A=\left\{a \in \mathbb{Z}^{d}: d_{E}\left(\Lambda_{a}, \Lambda_{b}\right)>r_{0}\right\}$ and $A^{c}$ is the complement in $\mathbb{Z}^{d}$ of $A$. By (3.16) the first sum in (3.21) is bounded by

$$
4 \beta z M(z, \beta) \sup _{b} \sum_{a \in A} e^{d(a, b)} \psi\left[d_{E}\left(\Lambda_{a}, \Lambda_{b}\right)\right]
$$

By Condition 3.1 this can be made arbitrarily small by choosing $z$ or $\beta$ sufficiently small. 
By (3.1) and (3.14) and since $\varrho\left(s_{a}, t_{a}\right) \geqq 1$ for $s_{a} \neq t_{a}$, the second sum in (3.21) is less than $\eta$ for $z$ less than some constant $C_{k}$. Thus by choosing $z<C_{k}$ and $z$ or $\beta$ sufficiently small, $\alpha<\eta$. This completes the proof.

For the remaining results in this section, $V$ need not be a pair potential.

Theorem 3.5. Let $V$ be finite ranged and satisfy Condition 3.2 with $k=1$, and let $\eta>0$ be given. There exists $z_{0}>0$ such that for $z<z_{0}, \alpha<\eta$. The number $z_{0}$ depends on the range of $V$.

Proof. By (3.1) and (3.14) $\varrho_{a, b} \leqq 4 z M(z, \beta)$ for all $a, b \in \mathbb{Z}^{d}$. But for any given $a \in \mathbb{Z}^{d}$, $\varrho_{a, b}=0$ for all but finitely many $b \in \mathbb{Z}^{d}$, because $V$ has finite range. Thus $\alpha$ is a finite sum of terms of the form $e^{d(a, b)} \varrho_{a, b}$ and each of these terms is bounded by $4 z e^{d(a, b)} M(z, \beta)$. Hence by choosing $z$ less than some $z_{0}$ we can make $\alpha<\eta$. This completes the proof.

Lemma 3.7. Let $V$ satisfy Conditions 3.1 and 3.2 with $k=1$, and let $s$ and $t$ be any two configurations in $U$ which differ only on $\Lambda_{a}$. If $d_{E}\left(\Lambda_{a}, \Lambda_{b}\right)>r_{0}$ and $\phi_{N_{0}}$ is hard-core for some $N_{0} \geqq 2$, then

$$
\varrho_{a, b} \leqq 4 N_{1} \beta z M(z, \beta) \psi(r),
$$

where $N_{1}$ is an integer which depends only on $\phi_{N_{0}}$.

Proof. Let $s_{a}$ and $t_{a}$ have coordinates in $\mathbb{R}^{d}$ given by $s_{a}=\left(s^{1}, \ldots, s^{n}\right)$ and $t_{a}=\left(t^{1}, \ldots, t^{m}\right)$. Assume that $m \geqq n$ and that the coordinates of $s_{a}$ and $t_{a}$ are ordered so that

$$
\sum_{i=1}^{n} d_{E}\left(s^{i}, t^{i}\right)+m-n=\varrho_{1}\left(s_{a}, t_{a}\right)
$$

In what follows we will require $x \in X_{k}\left(\Lambda_{b}\right) \cap U$. Let the coordinates of $x$ be given by $x=\left(x^{1}, \ldots, x^{k}\right)$. By hypothesis $s$ and $t$ differ only in $\Lambda_{a}$. This allows us to write $V_{b}(x \mid \hat{s})-V_{b}(x \mid \hat{t})$ in an explicit form. To simplify notation, we let min denote $\min \{|x|, N-1\}$ in the following equation and throughout the rest of this proof.

$$
\begin{aligned}
V_{b}(x \mid \hat{s})-V_{b}(x \mid \hat{t})= & \sum_{N=2}^{|x|+n} \sum_{p=1}^{\min } \sum_{I_{p}} \sum_{J_{n}} \phi_{N}\left(x_{i_{1}}, \ldots, x_{i_{p}}, s_{j_{p+1}}, \ldots, s_{j_{N}}\right) \\
& -\phi_{N}\left(x_{i_{1}}, \ldots, x_{i_{p}}, t_{j_{p}+1}, \ldots, t_{j_{N}}\right) \\
& +\sum_{N=2}^{|x|+m} \sum_{p=1}^{\min } \sum_{I_{p}} \sum_{J_{m}^{\prime}} \phi_{N}\left(x_{i_{1}}, \ldots, x_{i_{p}}, t_{j_{p+1}}, \ldots, t_{j_{N}}\right)
\end{aligned}
$$

where $\sum_{I_{p}}$ denotes a sum over all subsets $\left\{i_{1}, \ldots, i_{p}\right\}$, with cardinality $p$, of the integers $1, \ldots,|x|, \sum_{J_{n}}$ denotes a sum over all subsets $\left\{j_{p+1}, \ldots, j_{N}\right\}$, with cardinality $N-p$, of the integers $1, \ldots, n$, and $\sum_{J_{m}^{\prime}}$ denotes a sum over all subsets $\left\{j_{p+1}, \ldots, j_{N}\right\}$ 
with cardinality $N-p$ of the integers $1, \ldots, m$, such that $j_{k}>n$ for at least one $k$ between $p+1$ and $N$. By Condition 3.1

$$
\begin{aligned}
\left|V_{b}(x \mid \hat{s})-V_{b}(x \mid \hat{t})\right| \leqq & \sum_{N=2}^{|x|+n} \sum_{p=1}^{\min } \sum_{I_{p}} \sum_{J_{n}} K_{n} \psi(r)\left[d_{E}\left(s_{j_{p+1}}, t_{j_{p+1}}\right)+\ldots+d_{E}\left(s_{j_{N}}, t_{j_{N}}\right)\right] \\
& +\sum_{N=2}^{|x|+m} \sum_{p=1}^{\min } \sum_{I_{p}} \sum_{J_{m}^{\prime}} K_{N} \psi(r)
\end{aligned}
$$

where $r=d_{E}\left(\Lambda_{a}, \Lambda_{b}\right)$. Let us examine the expression

$$
\sum_{I_{p}} \sum_{J_{N}} K_{N} \psi(r)\left[d_{E}\left(s_{j_{p+1}}, t_{j_{p+1}}\right)+\ldots+d_{E}\left(s_{j_{N}}, t_{j_{N}}\right)\right]
$$

in (3.26). For each $k=1, \ldots, n$, the term $d_{E}\left(s_{k}, t_{k}\right)$ occurs $\left(\begin{array}{c}|x| \\ p\end{array}\right)\left(\begin{array}{c}n-1 \\ N-p-1\end{array}\right)$ times in (3.27). Thus we can replace (3.27) by

$$
K_{N} \psi(r)\left(\begin{array}{c}
|x| \\
p
\end{array}\right)\left(\begin{array}{c}
n-1 \\
N-p-1
\end{array}\right) \sum_{k=1}^{n} d_{E}\left(s_{k}, t_{k}\right) .
$$

We can also bound the expression

$$
\sum_{I_{p}} \sum_{J_{m}^{\prime}} K_{N} \psi(r)
$$

in (3.26) by $\left(\begin{array}{c}|x| \\ p\end{array}\right)\left(\begin{array}{c}m \\ N-p\end{array}\right) K_{N} \psi(r)$. Combining this with (3.26) and (3.28) gives

$$
\begin{aligned}
\left|V_{b}(x \mid \hat{s})-V_{b}(x \mid \hat{t})\right| \leqq & \psi(r)\left[\sum_{k=1}^{n} d_{E}\left(s_{k}, t_{k}\right)\right] \sum_{N=2}^{|x|+n} K_{N} \sum_{p=1}^{\min }\left(\begin{array}{c}
|x| \\
p
\end{array}\right)\left(\begin{array}{c}
n-1 \\
N-p-1
\end{array}\right) \\
& +\psi(r)(m-n) \sum_{N=2}^{|x|+m} K_{N} \sum_{p=1}^{\min }\left(\begin{array}{c}
|x| \\
p
\end{array}\right)\left(\begin{array}{c}
m \\
N-p
\end{array}\right) .
\end{aligned}
$$

The coefficient $m-n$ is zero when $m=n$ and at least one otherwise, so that inequality in (3.29) is maintained. Now let

$$
Q^{1}(|x|)=\sum_{N=2}^{|x|+n} K_{N} \sum_{p=1}^{\min }\left(\begin{array}{c}
|x| \\
p
\end{array}\right)\left(\begin{array}{c}
n-1 \\
N-p-1
\end{array}\right)
$$

and

$$
Q^{2}(|x|)=\sum_{N=2}^{|x|+m} K_{N} \sum_{p=1}^{\min }\left(\begin{array}{c}
|x| \\
p
\end{array}\right)\left(\begin{array}{c}
m \\
N-p
\end{array}\right) .
$$

Recall that $x, s, t \in U$ and that $\Lambda_{a}, \Lambda_{b}$ are bounded subsets of $\mathbb{R}^{d}$. By the definitions of $U$ and $R_{A}^{-}$and since $\phi_{N_{0}}$ is hard-core for some $N_{0} \geqq 2$, it follows that each of $|x|$, $\left|s_{b}\right|$, and $\left|t_{b}\right|$ is bounded by some positive integer $M$. From this it follows that for some positive integer $N_{1}$,

$$
Q^{1}(|x|), Q^{2}(|x|) \leqq N_{1}
$$


Combining (3.30) with (3.24) and (3.29) gives

$$
\left|V_{b}(x \mid \hat{s})-V_{b}(x \mid \hat{t})\right| \leqq N_{1} \psi(r) \varrho_{1}\left(s_{a}, t_{a}\right),
$$

for all $x \in X\left(\Lambda_{b}\right) \cap U$.

Now since $d\left(\Lambda_{a}, \Lambda_{b}\right)>r_{0}$ and $s$ and $t$ are identical except on $\Lambda_{a}$, we can apply Lemma 3.5. Thus combining (3.31) with (3.15) gives

$$
\begin{gathered}
\int_{X\left(\Lambda_{b}\right)} \varrho(x, \emptyset)\left|\frac{\exp \left(-\beta V_{b}(x \mid \hat{s})\right)}{Z_{b}(s)}-\frac{\exp \left(-\beta V_{b}(x \mid \hat{t})\right)}{Z_{b}(t)}\right| v_{b}(d x) \\
\leqq 2 \beta \psi(r) N_{1} \varrho_{1}\left(s_{a}, t_{a}\right) \int_{0}^{1} \int_{X\left(\Lambda_{b}\right)} \varrho(x, \emptyset) d \mu_{\lambda} d \lambda .
\end{gathered}
$$

Since $\varrho(x, \emptyset) \leqq 2|x|$ we can apply Condition 3.2 to get

$$
\int_{x\left(\Lambda_{b}\right)} \varrho(x, \emptyset)\left|\frac{\exp \left(-\beta V_{b}(x \mid \hat{s})\right)}{Z_{b}(s)}-\frac{\exp \left(-\beta V_{b}(x \mid \hat{t})\right)}{Z_{b}(t)}\right| v_{b}(d x) \leqq 4 N_{1} \beta z M(z, \beta) \varrho_{1}\left(s_{a}, t_{a}\right) \psi(r) .
$$

Combining this with (3.1) gives

$$
\varrho_{a, b} \leqq 4 N_{1} \beta z M(z, \beta) \psi(r) .
$$

This completes the proof.

Corollary 3.1. With the same conditions as in Lemma 3.7, except that $V$ does not satisfy part c) of Condition 3.1 we have

$$
\varrho_{a, b} \leqq 4 N^{1} \beta z M(z, \beta) \psi(r),
$$

where $N^{1}$ is a positive integer which depends only on $\phi_{N_{0}}$.

Proof. The proof begins exactly as in the proof of Lemma 3.7 up to (3.26). Now replace (3.26) with

$$
\left|V_{b}(x \mid \hat{s})-V_{b}(x \mid \hat{t})\right| \leqq \sum_{N=2}^{|x|+n} \sum_{p=1}^{\min } \sum_{I_{p}} \sum_{J_{n}} 2 K_{N} \psi(r)+\sum_{N=2}^{|x|+m} \sum_{p=1}^{\min } \sum_{I_{p}} \sum_{J_{m}^{\prime}} K_{N} \psi(r) .
$$

Noting the remarks preceding (3.30) that $|x|,\left|s_{b}\right|$, and $\left|t_{b}\right|$ are bounded by some integer $K$, we can conclude that $\left|V_{b}(x \mid \hat{s})-V_{b}(x \mid \hat{t})\right| \leqq N^{1} \psi(r)$ for all $x \in X\left(\Lambda_{b}\right) \cap U$ and some positive integer $N^{1}$. The argument now proceeds as in the final paragraph of the proof of Lemma 3.7. This completes the proof.

Remark 3.2. We note in comparing Lemma 3.7 with Corollary 3.1 that $N^{1}$ is generally larger than $N_{1}$.

We conclude this section with a theorem for many-body potentials whose proof is exactly analogous to the proof of Theorem 3.4.

Theorem 3.6. Suppose that $V$ satisfies Condition 3.2 with $k=1$ and parts a) and b) of Condition 3.1, and that $\phi_{N_{0}}$ is hard-core for some $N_{0} \geqq 2$. Let $K>0$ and $\eta>0$ be given. If $z, \beta<K$ then there exists $C_{k}>0$ such that if $z<C_{k}$, then $\alpha<\eta$ for $\beta$ or $z$ sufficiently small. 


\section{Section 4. Summary}

In Sect. 3 we found conditions on a potential $V$ so that : a) $\tau_{A}: F(U) \rightarrow F(U)$, b) any Gibbs state for $V$ (if one exists) satisfies (2.8), and c) $V$ satisfies (2.7) and (2.9), for high temperature or low activity. Let $H$ denote the set of all stable, $S$-measurable, translation-invariant potentials which satisfy a), b), and c) above. The results of Sect. 2 give :

Theorem 4.1. Let $d(\cdot, \cdot)$ be a translation invariant semi-metric on $\mathbb{Z}^{d} \subset \mathbb{R}^{d}$, and let $K_{1}>0$ be given. If $V \in H$ and $a$ Gibbs state $\sigma$ exists for $V$ when $z, \beta<K_{1}$, then there exists $K_{2}>0$ such that if $z<K_{2}$ and $z$ or $\beta$ is sufficiently small, the following conditions hold:

$$
\begin{aligned}
& \sigma \text { is unique, } \\
& |\sigma(f g)-\sigma(f) \sigma(g)| \leqq C e^{-d(a, b)}\|f\|_{a}\|g\|_{b}(1-\alpha)^{-2}\left(1-\alpha^{2}\right)^{-1} \\
& \text { for some constant } C \text {, all } a, b \in \mathbb{Z}^{d} \text {, and all } f, g \in L(U) \text {, } \\
& \sum_{a \in \mathbb{Z}^{d}}\left|\sigma\left(f g_{a}\right)-\sigma(f) \sigma\left(g_{a}\right)\right| e^{d(a, c)} \leqq C\|f\|_{c}\|g\|_{0}(1-\alpha)^{-1}\left(1-\alpha-\alpha^{2}\right)^{-1} \\
& \text { for the same constant } C \text { as in }(4.2), \text { all } c \in \mathbb{Z}^{d} \text {, and } f, g \in L(U) \text {. }
\end{aligned}
$$

Remark 4.1. We have not shown the existence of the Gibbs state $\sigma$. Ruelle [14] (see also Preston [12]) has proven existence for a wide class of pair potentials, though we know of no such result for many-body potentials.

Remark 4.2. The restriction that $f, g \in L(U)$ can be somewhat relaxes. For example, let $N^{a}(s)=\left|s \cap \Lambda_{a}\right|$ for any $a \in \mathbb{Z}^{d}$ and $s \in U$. The function $N^{a}(s)$ gives the number of particles in the configuration $s$ which lie in the cube $\Lambda_{a}$. Since $N^{a}$ is unbounded, $N^{a} \notin L(U)$. However, we can apply (4.2) and (4.3) in this case in the following manner. Let

$$
f_{n}^{a}(s)= \begin{cases}N^{a}(s) & \text { if }\left|s \cap \Lambda_{a}\right| \leqq n \\ n & \text { otherwise }\end{cases}
$$

It is not hard to check that $f_{n}^{a} \in L(U)$ and that $\left\|f_{n}^{a}\right\|_{a} \leqq 1$ for $n \geqq 1$. Then by the monotone convergence theorem we have from (4.2),

$$
\left|\sigma\left(N^{a} N^{b}\right)-\sigma\left(N^{a}\right) \sigma\left(N^{b}\right)\right| \leqq C e^{-d(a, b)}(1-\alpha)^{-1}\left(1-\alpha^{2}\right)^{-1}
$$

With 0 referring to the origin in $\mathbb{Z}^{d}$, the monotone convergence theorem together with Fatou's lemma and (4.3) give

$$
\sum_{a \in \mathbb{Z}^{d}}\left|\sigma\left(N^{c} N_{a}^{0}\right)-\sigma\left(N^{c}\right) \sigma\left(N_{a}^{0}\right)\right| e^{d(a, c)} \leqq C(1-\alpha)^{-1}\left(1-\alpha-\alpha^{2}\right)^{-1} .
$$

In a similar fashion we can apply (4.2) and (4.3) to functions of the type $N^{\Lambda}(s)=|s \cap \Lambda|$, where $\Lambda$ is a bounded Borel set in $\mathbb{R}^{d}$. For a clear discussion of the physical significance of the averaged correlations $\sigma\left(N^{\Lambda} N^{\tilde{\Lambda}}\right)-\sigma\left(N^{\Lambda}\right) \sigma\left(N^{\tilde{\Lambda}}\right)$ and how they are related to the more standard "non-averaged" correlation functions, we refer the reader to Minlos [11]. 
Remark 4.3. Comparing results of Sect. 3 yields the following sufficient condition for $V \in H$. A stable, translation invariant, $S$-measurable potential $V$ is an element of $H$ if each of the following conditions holds:

a) $V$ satisfies Condition 3.2 with $k=2$ and parts a) and b) of Condition 3.1 given in Sect. 3.

b) Either $V$ has finite range or $V$ satisfies all of the following:

1) $\phi_{N}\left(x^{1}, \ldots, x^{N}\right)=0$ whenever $\max _{i j} d_{E}\left(x^{i}, x^{j}\right)>R_{0}$ for some given $R_{0}>0$ and all $N \geqq 3$.

2) $\phi_{N_{0}}$ is hard-core for some $N_{0} \geqq 2$.

We make a final observation about the above remark. The condition that $\phi_{N_{0}}$ is hard-core for some $N_{0} \geqq 2$ can be considered in the following way. The main technical problem in establishing conditions for $V \in H$ has been in dealing with the low probability event that some huge number of particles cluster in some small region of space. The methods of proof in this paper do not seem to allow for exploitation of the small probability of this event. However, in dealing with a potential $V$ of the form

$$
V(x)=\sum_{N=2}^{M} \sum_{\substack{y \subset x \\|y|=N}} \phi_{N}(y)
$$

where $M$ is finite, we can add to $V$ the $N_{0}$-body potential defined by

$$
\phi_{N_{0}}(x)= \begin{cases}\infty & \text { if } \max _{i j} d_{E}\left(x^{i}, x^{j}\right)<R_{0} \\ 0 & \text { otherwise }\end{cases}
$$

where $R_{0}$ is very small and $N_{0}$ is large. This has the effect of assigning zero probability to the event that $N_{0}$ or more particles accumulate in a spherical region of space of diameter $R_{0}$. Thus, for example, in dealing with a non-hard-core pair potential $V$, we could add $\phi_{N_{0}}$ to $V$ as defined in (4.4) with $N_{0}=10^{23}$ and $R_{0}=1$ angstrom. In this fashion the physically unreasonable event that $10^{23}$ or more particles accumulate in such a small region of space is assigned zero probability, even though any smaller number of particles has some non-zero probability of clustering in any region of space. We could, in this manner, apply the results of this paper to a pair potential $V \notin H$, but satisfying Condition 3.2 and parts a) and b) of Condition 3.1. The potential $V$ modified as above is then an element of $H$. (We point out, however, that some non-hard-core pair potentials are elements of $H$ without such modifications.)

A drawback to this approach is that the larger the number $N_{0}$, for a fixed value of $R_{0}$, the smaller the values of $z$ and $\beta$ must be (with the present techniques employed in this paper) to guarantee uniqueness of the Gibbs state, and the decay of correlations. This problem should be somewhat mitigated if, in all of the preceding analysis, $\mathbb{Z}^{d}$ is replaced by $\lambda \mathbb{Z}^{d}$ for some small $\lambda>0$, and the function $\psi(r)$ of Condition 3.1 is required to fall of very rapidly (depending on $N_{0}$ and $R_{0}$ ) as $r$ approaches infinity.

Acknowledgement. The author is deeply indebted to L. Gross for his generous assistance and numerous suggestions. 


\section{References}

1. Dobrushin, R.L.: The description of a random field by means of conditional probabilities and conditions on its regularity. Theor. Probab. Appl. 13, 197-224 (1968)

2. Dobrushin, R.L.: Prescribing a system of random variables by conditional distributions, Theor. Probab. Appl. 15, 458-486 (1970)

3. Duneau, M., Souillard, B.: Cluster properties of lattice and continuous systems. Commun. Math. Phys. 47, 155-166 (1976)

4. Duneau, M., Souillard, B., Iagolnitzer, D.: Decay of correlations for infinite-range interactions. J. Math. Phys. 16, 1662-1666 (1975)

5. Iagolnitzer, D., Souillard, B.: Decay of correlations for slowly decreasing potentials. Phys. Rev. A16, 1700-1704 (1977)

6. Gross, L. : Decay of correlations in classical lattice models at high temperature. Commun. Math. Phys. 68, 9-27 (1979)

7. Gross, L.: Absence of second order phase transitions in the Dobrushin uniqueness region. J. Stat. Phys. 25, 57-72 (1981)

8. Gross, L.: Thermodynamics, statistical mechanics and random fields. In: Lecture Notes in Mathematics. Berlin, Heidelberg, New York: Springer 1982 (to appear)

9. Lanford, O.E., III : Entropy and equilibrium states in classical statistical mechanics. In: Lecture Notes in Physics, Vol. 20. Statistical mechanics and mathematical problems. Lenard, A. (ed.). Berlin, Heidelberg, New York: Springer 1973

10. Lanford, O.E., III, Ruelle, D.: Observables at infinity and states with short range correlations in statistical mechanics. Commun. Math. Phys. 13, 194-215 (1969)

11. Minlos, R.A. : Lectures on statistical physics. Russ. Math. Surv. 23, 137-194 (English translation) (1968)

12. Preston, C.: Random fields. In: Lecture Notes in Mathematics, Vol. 534. Berlin, Heidelberg, New York: Springer 1976

13. Ruelle, D.: Statistical mechanics. New York: Benjamin 1969

14. Ruelle, D.: Superstable interactions in classical statistical mechanics. Commun. Math. Phys. 18, 127-159 (1970)

15. Ruelle, D.: Thermodynamic formalism. Reading, Mass.: Addison Wesley 1978

16. Simon, B.: A remark on Dobrushin's uniqueness theorem. Commun. Math. Phys. 68, 183-185 (1979)

17. Vasershtein, L.N. : Markov process over denumerable products of spaces, describing large systems of automata. Prob. Transm. Inform. 5, 64-72 (English translation) (1969)

Communicated by E. Lieb

Received March 31, 1982 